Research Paper

\title{
HJURP Promotes Epithelial-to-Mesenchymal Transition via Upregulating SPHK1 in Hepatocellular Carcinoma
}

\author{
Tianchi Chen ${ }^{1,2,3,4,5^{*}}$, Lingfeng Zhou1 1,2,3,4,5*, Yuan Zhou 2,3,4,5, Wuhua Zhou 2,3,4,5, Hechen Huang 2,3,4,5, \\ Shengyong Yin 2,3,4, Haiyang Xie $2,3,4,5$, Lin Zhou $2,3,4,5$, Shusen Zheng $1,2,3,4,5 \bowtie$ \\ 1. Division of Hepatobiliary and Pancreatic Surgery, Department of Surgery, First Affiliated Hospital, School of Medicine, Zhejiang University, Hangzhou, \\ China. \\ 2. NHFPC Key Laboratory of Combined Multi-organ Transplantation, Hangzhou, China. \\ 3. Key Laboratory of Organ Transplantation, Zhejiang Province, Hangzhou, China. \\ 4. Key Laboratory of the diagnosis and treatment of organ Transplantation, CAMS, Hangzhou, China. \\ 5. Collaborative innovation center for Diagnosis treatment of infectious diseases, Zhejiang university, Hangzhou, China. \\ *These authors contributed equally to this work.
}

$\square$ Corresponding author: Prof. Shusen Zheng. E-mail: shusenzheng@zju.edu.cn; Tel: 0571-87236466; Address: Division of Hepatobiliary and Pancreatic Surgery, Department of Surgery, First Affiliated Hospital, School of Medicine, Zhejiang University, 79 QingChun Road, Hangzhou 310003, China.

(1) Ivyspring International Publisher. This is an open access article distributed under the terms of the Creative Commons Attribution (CC BY-NC) license (https://creativecommons.org/licenses/by-nc/4.0/). See http://ivyspring.com/terms for full terms and conditions.

Received: 2019.03.14; Accepted: 2019.04.07; Published: 2019.05.07

\begin{abstract}
Holliday Junction Recognition Protein (HJURP) is involved in various cancers including hepatocellular carcinoma (HCC). Current studies have showed that HJURP is correlated with HCC proliferation. However, the role of HJURP in HCC Epithelial-to-Mesenchymal Transition remains unclear. In this study, we found that HJURP knockdown significantly reduced the migration and invasion abilities of HCC cells both in vivo and in vitro by interacting with Sphingosine kinasel (SPHK1). Conversely, HJURP overexpression enhanced these biological abilities. Moreover, high HJURP expression is related to poor prognosis of HCC patients. In conclusion, HJURP plays an important role in tumor metastasis by upregulating SPHK1. And high HJURP expression may predict a lower disease-free survival rate and higher possibility of microvascular invasion in HCC patients.
\end{abstract}

Key words: Holliday junction recognition protein, epithelial-to-mesenchymal transition, sphingosine kinase 1, hepatocellular carcinoma

\section{Introduction}

Hepatocellular carcinoma (HCC) is one of the most common malignancies worldwide[1]. According to some epidemiologic studies, around 780,000 new patients were diagnosed worldwide in 2012 and nearly 750,000 cases of HCC-related death were reported, which makes it the third leading cause of cancer-related death in the world[2]. With the development of medical technology, early diagnosis and advanced therapeutic approaches have improved survival rates of HCC individuals[3]. However, due to lack of early symptoms, HCC is usually diagnosed at advanced stage and its therapeutic options are limited, in which cases, patients generally die within a few years of diagnosis. Thus, it is essential to illuminate the molecular mechanisms underlying tumorigenesis and metastasis in HCC and find novel therapeutic targets for HCC patients[4].

Holliday Junction Recognition Protein (HJURP) is identified as a sort of centromeric protein that plays a pivotal role in the assembly and maintenance of histone H3-like variant CENP-A[5], and it helps the recruitment of CENP-C and mediates chromosome segregation and cell division[6]. It has been reported to have a significantly higher expression in HCC tissues compared to non-tumor tissues[7, 8]. Overexpression of HJURP accelerates HCC proliferation in vitro and it leads to an unfavorable clinical outcome in hepatocellular carcinoma[7]. In addition, Huang et. al. indicated that SNP of HJURP, rs3771333 plays an important role in mediating the susceptibility to HCC in Chinese population[9]. However, there are no 
studies illuminating the mechanism of HJURP in tumor metastasis.

Epithelial-to-Mesenchymal Transition (EMT), known as the ability of epithelial cells to transdifferentiation into mesenchymal cells, plays an important role in embryonic development, tissue repair, organ fibrosis and cancer progression[10-12]. During the progress of EMT, epithelial cells reorganize their cortical actin cytoskeleton, undergo a change in cell shape, decrease cell-cell adhesion and increase the motility of individual cells, which enables the development of an invasive phenotype[13]. Generally, EMT is mediated by key factors, including SNAIL, zinc-finger E-box-binding (ZEB), lymphoid enhancer binding factor-1 (LEF-1) and basic helix-loop-helix transcription factors[14, 15]. Besides, Sphingosine kinase1 (SPHK1) can transform sphingosine to sphingosine 1-phosphate, which has been reported to play an important role in regulating EMT in human neoplasms[16]. Xu et.al. showed that SPHK1 modulates the expression of EMT-related markers and cell migration by regulating the expression of p-FAK in colorectal cancer cells[17]. Liu et. al. demonstrated that SPHK1 induces EMT by facilitating E-cadherin lysosomal degradation in HCC cells[18]. Although SPHK1 has been demonstrated to be important in EMT, the role of SPHK1 in HJURPinduced EMT remains to be clarified.

Herein, we investigated the role of HJURP involved in HCC metastasis and analyzed the relation between HJURP expression and clinicopathological features.

\section{Materials and Methods}

\section{Lentivirus vector information}

The lentivirus of HJURP and SPHK1 were constructed in Genechem Company (Shanghai, China). HJURP and SPHK1 overexpression vector: Ubi-MCS3FLAG-SV40-IRES-puromycin. HJURP knockdown vector: hU6-MCS-Ubiquitin-IRES-puromycin. Transfection of lentivirus was based on manufacturer's instruction book.

\section{Cell migration and invasion assays}

Cell migration and invasion assay were performed by Millicell Cell Culture Inserts $(8 \mu \mathrm{m}$ pore size, Millipore, USA). Briefly, $5 \times 10^{4}$ cells were seeded in serum-free medium into upper insert with (invasion assay) or without (migration assay) Matrigel Matrix (BD, USA). The subjacent compartment was added with $10 \%$ FBS medium. After incubation for $48 \mathrm{~h}$, cells on the lower surface of insert were fixed with $4 \%$ paraformaldehyde and stained using $0.5 \%$ crystal violet solution.

\section{Western-blot}

All cell lysates were extracted with RIPA (Cell Signaling Technology, USA) and proteinase inhibitor (Cell Signaling Technology, USA), which were mixed in advance. The supernatant was collected from samples after centrifugation at $12,000 \mathrm{~g}$ for $15 \mathrm{~min}$. Protein concentrations were measured using the BCA protein assay kit (Thermo Scientific, USA) according to the manufacturer's instructions. Equal amounts of protein (30ug) were subjected to $10 \%$ sodium dodecyl sulfate-polyacrylamide gel electrophoresis (SDSPAGE) (Thermo Scientific, USA) and transferred to polyvinylidene difluoride (PVDF) membranes (Millipore, USA). Membranes were blocked with Trisbuffered saline plus Tween 20 (TBST; 0.1\% Tween-20) containing $5 \%$ non-fat dry milk at room temperature and incubated with corresponding primary antibodies overnight at $4^{\circ} \mathrm{C}$. Primary antibodies include mouse anti-human GAPDH monoclonal antibody, HJURP (Abcam, UK), E-Cadherin, N-cadherin, Vimentin, and SPHK1 (Cell Signaling Technology, USA). After washing with TBST, the membranes were incubated with horseradish peroxidase-labeled secondary antibodies $(1: 5000)$ for $1 \mathrm{~h}$ at room temperature. The membranes were detected by enhanced chemiluminescence method (Thermo scientific, USA).

\section{Co-Immunoprecipitation (Co-IP)}

Cell samples were lysed in a IP lysis buffer containing $1 \mathrm{mM} \mathrm{MgCl}, 100 \mathrm{mmol} / \mathrm{L} \mathrm{NaCl}, 1 \mathrm{mM}$ DL-dithiothreitol (DTT) (Life Technology, USA) and protease inhibitor cocktails (Cell Signaling Technology, USA). The homogenates were incubated on ice for 30-45 min. Subsequently, cell samples were centrifuged at $2600 \mathrm{~g}$ for $15 \mathrm{~min}$ at $4^{\circ} \mathrm{C}$. The total cell lysates were used for immunoprecipitation with HJURP and SPHK1 primary antibodies on protein A/G mix beads (Thermo Scientific, USA) overnight. After overnight incubation, the immunoprecipitates were collected and washed three times and prepared for western-blot analysis.

\section{RT-qPCR}

Total RNA was extracted from HCC cell lines or tissue samples using Trizol Reagent (Life, USA) according to the manufacturer's instruction. To analysis the expression of mRNA, total RNA was reverse transcribed to cDNA using Prime Script RT master mix (Takara, Japan), after which Quantitative Real Time-PCR (RT-qPCR) was performed with SYBR Premix Ex Taq (Takara, Japan). It was calculated by using the 2- $\triangle \mathrm{CT}$ method and GAPDH was taken as the endogenous control. The relative expression of HJURP between HCC cell lines was calculated using 
the $2-\Delta \Delta C T$ method. The sequences of primers used were listed in Table 1.

Table 1. The sequences of primers.

\begin{tabular}{lll}
\hline Gene & & Sequence of primers $\left(\mathbf{3}^{\prime}-\mathbf{5}^{\prime}\right)$ \\
\hline HJURP & Forward & AGTGCCTTATGTATTGGAG \\
& Reverse & AAGTGAGGGTCTGGATTTA \\
SPHK1 & Forward & CATCACGGCCTGTAAAAAGGT \\
& Reverse & ATCTTCCACAAACCCAATCTGG \\
GAPDH & Forward & GAACATCATCCCTGCCTCTACT \\
& Reverse & ATTTGGCAGGTTTTCTAGACG \\
\hline
\end{tabular}

\section{Immunofluorescence assay}

For immunofluorescence assay, $5 \times 10^{4}$ stable transfected tumor cells were seeded in $2 \mathrm{~mm}$ confocal plate (Nunc, USA) for culture in indicated incubator. Cultured cells were fixed with $4 \%$ paraformaldehyde and then permeabilized by $0.25 \%$ TritonX-100. After blocking with $1 \%$ bovine serum albumin (BSA), primary antibodies against E-cadherin (1:50) and Vimentin (1:100) (Cell Signaling Technology, USA) were added into each plate for an overnight incubation at $4^{\circ} \mathrm{C}$. A secondary antibody (1:200, Sigma-Aldrich, USA) was incubated at $37^{\circ} \mathrm{C}$ for 30 min and DAPI was used to stain the nuclei at room temperature for $15 \mathrm{~min}$. The images were captured by a fluorescence microscope (Olympus BX53, Japan).

\section{In vivo metastasis assays}

To establish in vivo metastasis assays, $1 \times 10^{6}$ tumor cells per mouse were suspended in $50 \mu$ PBS and injected via tail vein. After 10 weeks, liver and lung were harvested for H\&E staining and calculation of metastasis lesions.

\section{Statistical analysis}

The data are presented as mean \pm SD. Comparisons between two groups were analyzed by Student's $t$ test. The overall survival rate (OS) and disease free survival rate (DFS) curves of HCC patients based on Kaplan-Meier method were plotted using the log-rank test. The correlations between HJURP and clinicopathological features were assessed with $\chi^{2}$ tests. $P$ values $<0.05$ were considered statistically significant, * represents $P<0.05$, ** represents $P<0.01$ and ${ }^{* * *}$ represents $P<0.001$.

\section{Results}

\section{The expression of HJURP in HCC cell lines and tissues}

For the role of HJURP in HCC, we examined the expression of HJURP in HCC cell lines and clinical tissues. Data from Oncomine database revealed that mRNA of HJURP increased in HCC tissues (Figure 1A). Western-blot showed that HJURP remarkably overexpressed in five HCC cell lines compared to an immortalized hepatic cell line (L02) (Figure 1B). In addition, immunohistochemistry (IHC) staining and western-blot revealed that HJURP was significantly higher in HCC tissues than that in adjacent normal tissues (Figure 1C, 1F). Then, HCC-LM3 and Huh7 were used to construct HJURP stable transfected HCC cell lines. And the efficiency of HJURP-silenced and HJURP-overexpressed HCC cell lines were confirmed by western-blot (Figure 1D).

\section{HJURP promotes cell migration and invasion by activating Epithelial-to-Mesenchymal Transition in vitro and facilitates tumor metastasis in vivo}

For more biological functions of HJURP, transwell assay was used to validate the migration and invasion capabilities of HCC cells. HJURP knockdown dramatically suppressed Huh7 and HCC-LM3 cells invasion and migration. Inversely, overexpression of HJURP in Huh7 and HCC-LM3 cells promoted its invasion and migration (Figure 2A and B, Supplementary Figure 1A, 1B). Moreover, we established an in vivo tumor metastasis model to study the effect of HJURP on pulmonary metastasis. In consistent with results of in vitro experiments, HJURP down-expression HCC-LM3 cells caused less lung metastasis clusters than its control group (Figure 2C). Subsequently, we studied EMT proteins to understand the mechanism in relation to HJURP mediated aggressive effect. And E-cadherin was significantly upregulated whereas Vimentin were downregulated in HJURP-knockdown HCC-LM3 cells. And the same results were mirrored in HJURP overexpression group (Figure 2D). Immunofluorescence assay revealed that HJURP silence significantly enhanced the expression of E-cadherin and inhibited the expression of vimentin. Besides, HJURP overexpression in Huh7 cells resulted in E-cadherin decrease and vimentin enhancement (Figure 2E).

\section{HJURP facilitates EMT via upregulating Sphingosine Kinase 1}

To further investigate the mechanism of HJURP induced EMT, we performed a microarray analysis in Huh7 cells (Figure 3A). Based on the results of microarray, 85 genes were upregulated and 79 genes were downregulated. In those 164 differential expressed genes, 20 EMT-related genes were tested by RT-qPCR. Dramatically, only Sphingosine Kinase 1 (SPHK1) changed with HJURP knockdown or overexpression (Figure 3B). And the KEGG pathway analysis proved that HJURP regulated sphingosine metabolites process (Figure 3C). According to this result, we wondered whether HJURP induced EMT 
via SPHK1. To address that hypothesis, we detected the expression of SPHK1 in HJURP knockdown and overexpression cells respectively. Western-blot showed that HJURP silence inhibited the expression of SPHK1. Conversely, HJURP overexpression increased the expression of SPHK1 (Figure 3E). Moreover, SPHK1 overexpression significantly reversed the invasion abilities and EMT markers (E-cadherin, Vimentin and N-cadherin) in HJURPknockdown Huh7 and HCC-LM3 cells (Figure 4A and B). To further elucidate whether HJURP interacts with SPHK1, Co-IP assay was performed to detect endogenous HJURP and SPHK1 in HCC-LM3 cells. As shown in Figure 3F, HJURP was found to be co-precipitated with SPHK1.

\section{The expression of HJURP is positively correlated with SPHK 1}

We next determined the correlation between HJURP and SPHK1. Data from The Cancer Genome Atlas (TCGA) database showed that mRNA of SPHK1 was correlated with that of HJURP (Figure 3D). Furthermore, immunohistochemistry of HCC tissues revealed that $72.86 \%$ HCC specimens with high HJURP expression were accompanied with high SPHK1 expression. Similarly, $71.43 \%$ of samples with low HJURP expression displayed weak staining of SPHK1 (Figure 5A and B).
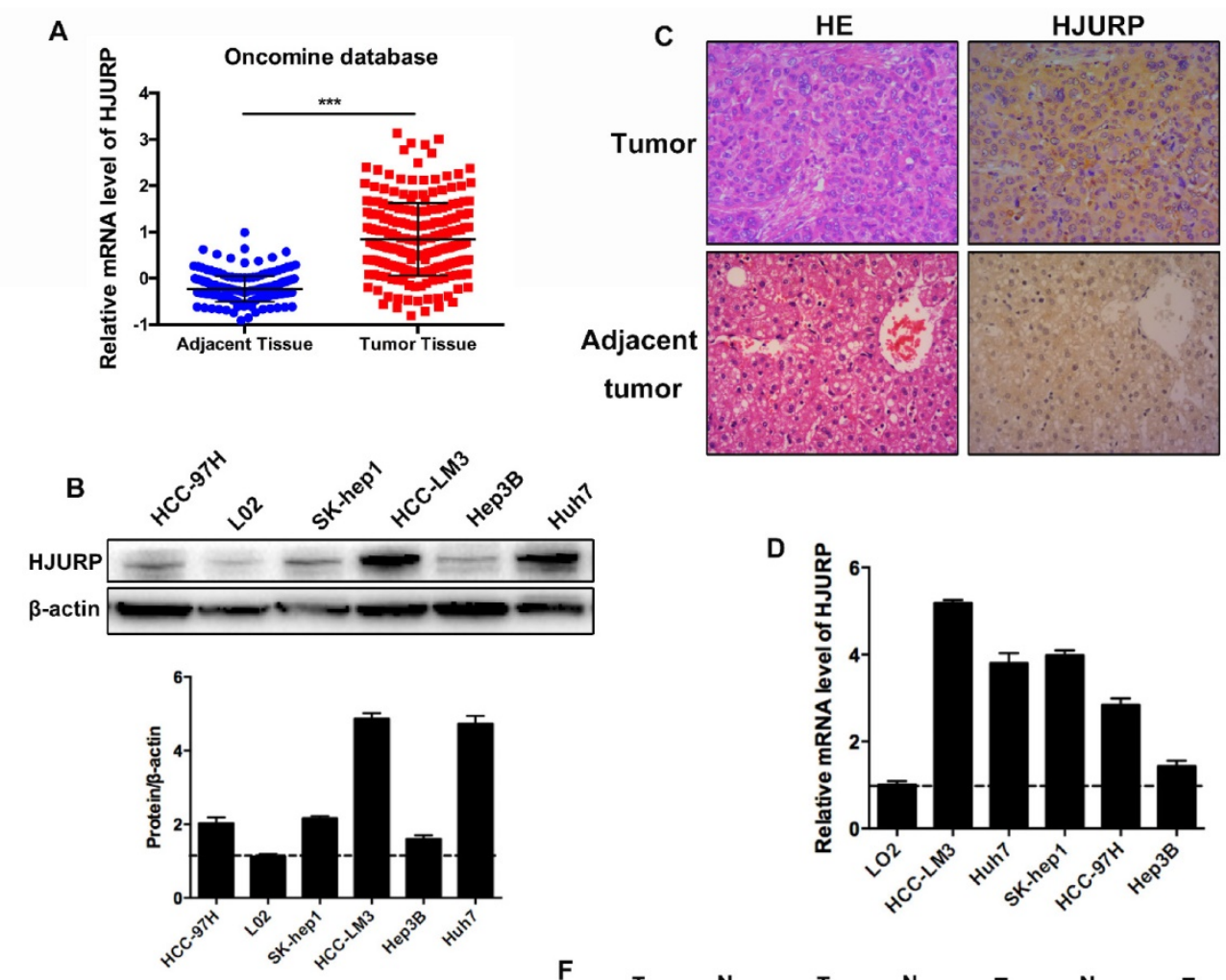

D
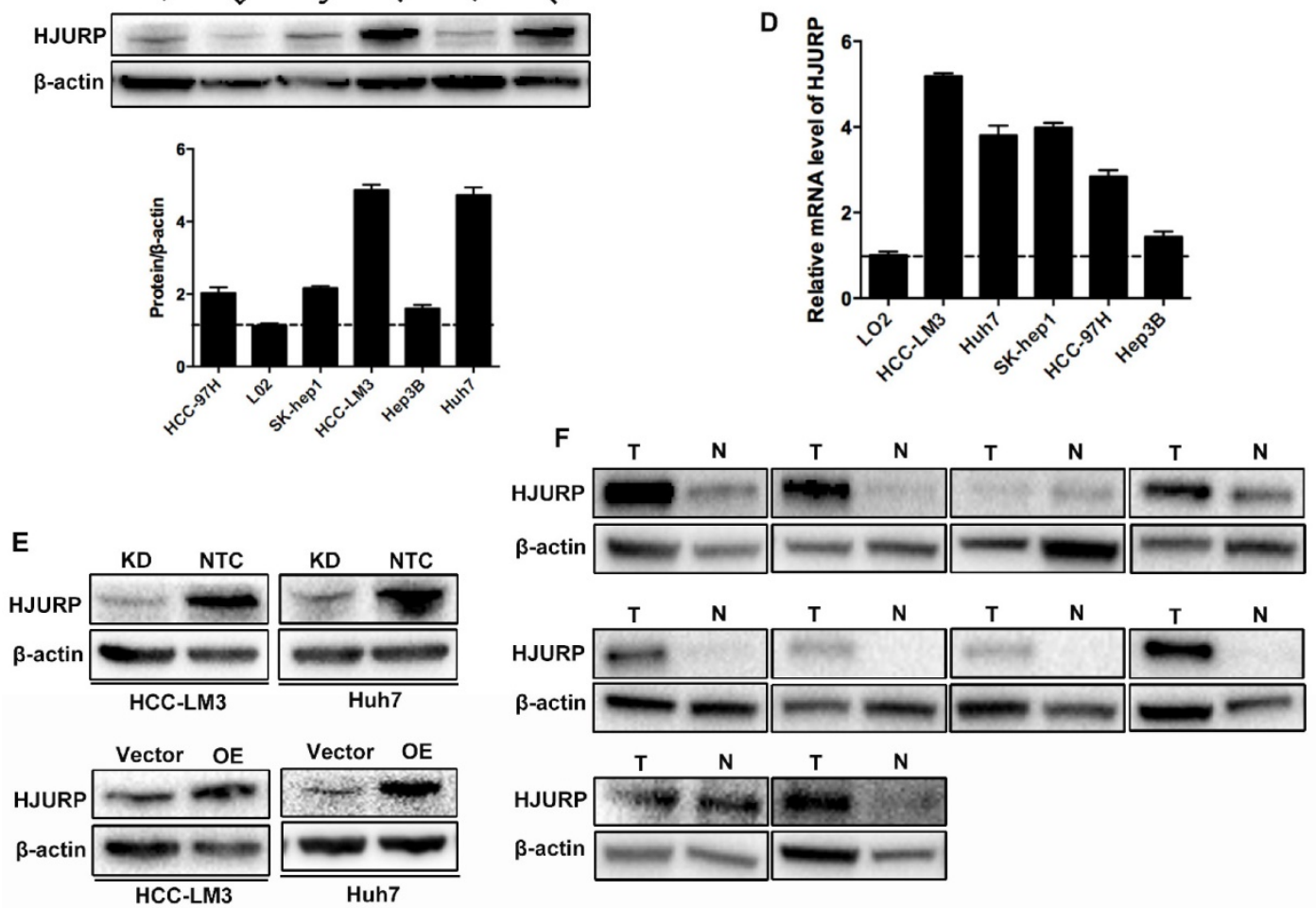

Figure 1. HJURP is highly expressed in HCC tissues and cell lines. (A) mRNA level of HJURP in Oncomine database. (B) Western-blot analysis of HJURP in five different HCC cell lines and one immortalized hepatic cell line (LO2). (C) Immunohistochemistry staining of HCC tissues and adjacent normal tissues (magnification: 200X). (D)Relative mRNA level of HJURP in five different HCC cell lines and one immortalized hepatic cell line. (E) The efficiencies of HJURP knockdown and overexpression were detected by western-blot. (F) HJURP was detected by western-blot in 10 pairs of HCC and adjacent tissues (T: tumor tissues; $\mathrm{N}$ : normal tissues). 


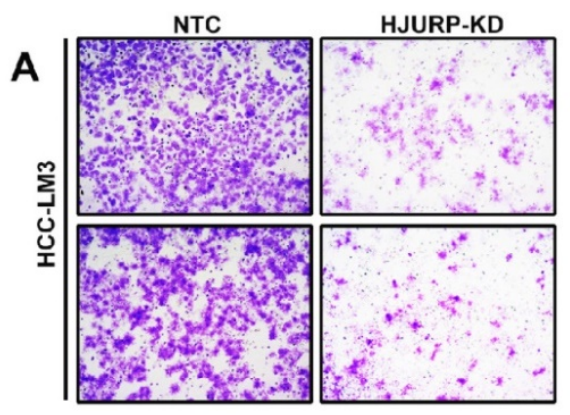

B
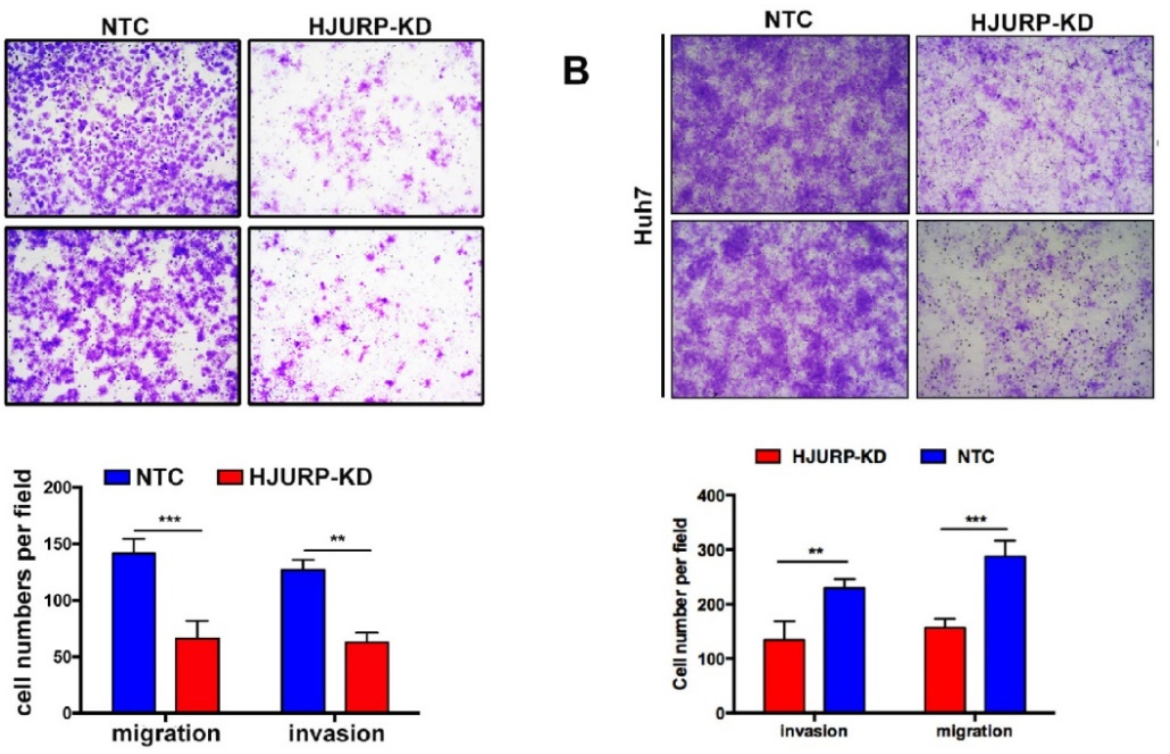

C
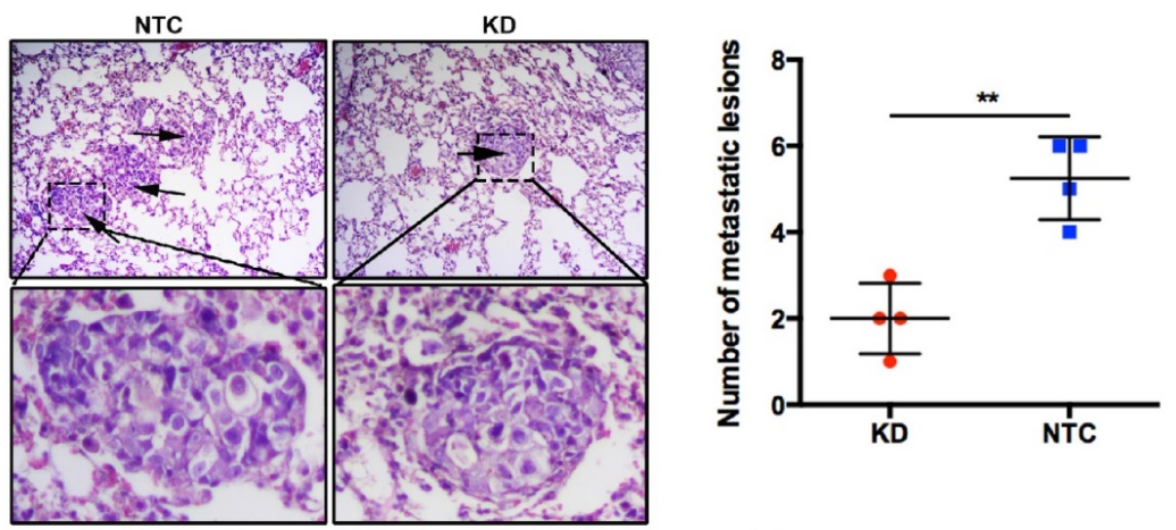

E
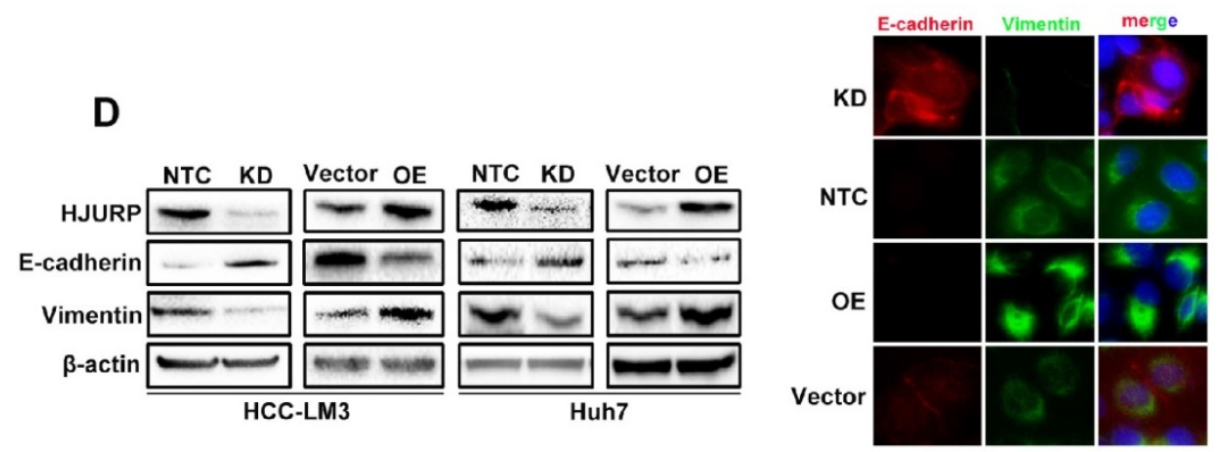

Figure 2. HJURP promotes cell migration and invasion by activating Epithelial-to-Mesenchymal Transition in vitro and facilitates tumor metastasis in vivo. (A) The invasion and migration abilities were significantly decreased in HJURP-knockdown group (HJURP-KD) HCC-LM3 and Huh7(B) cells compared to the control group (NTC). (C) Representative pictures H\&E staining from HJURP-KD and NTC group ( $n=4)$. Lung metastasis lesions were shown by black arrows. (D)EMT related protein (E-cadherin, Vimentin) were detected by western-blot. (E) E-cadherin (red) and Vimentin (green) were analyzed by immunofluorescence in HCC-LM3 cells (HJURP-knockdown) and Huh7 cells (HJURP-overexpression).

\section{High HJURP expression predicts poor clinicopathological features of HCC patients}

We previously reported HCC patients with high HJURP expression showed lower overall survival rate than that with low HJURP expression[8]. In this study, we performed analysis between HJURP expression and some clinicopathological features (Table 2). We found that high HJURP expression prompted a lower disease-free survival rate and more microvascular invasion in HCC patients (Figure 5C and Table 2). Besides, the multivariable cox analysis revealed that HJURP is an independent risk factor of tumor recurrence(Table 3 ). 


\section{Discussion}

HJURP has been demonstrated to be correlated with different kinds of cancer including HCC. According to current studies, HJURP acts as an oncogene in HCC $[7,8]$. Although HJURP has been reported to mediate HCC proliferation, HJURPinduced metastasis has not yet been studied.

In this study, we demonstrated that HJURP was significantly overexpressed both in HCC cell lines and clinical specimens. And HJURP promoted HCC cell migration and invasion in vitro and facilitates HCC cells metastasis in vivo. According to current reports, EMT is one of the major causes of cancer metastasis [19]. And a hallmark of EMT is the downregulation of E-cadherin, accompanied with the upregulation of mesenchymal markers, such as $\mathrm{N}$-cadherin and Vimentin[20], which is called 'cadherin switch' to alters cell-cell adhesion[21, 22]. In our study, HJURP knockdown suppressed HCC cell migration and invasion, led to upregulation of E-cadherin and downregulation of $\mathrm{N}$-cadherin and Vimentin. Thus, we revealed that HJURP promotes HCC migration and invasion through facilitating EMT.
A

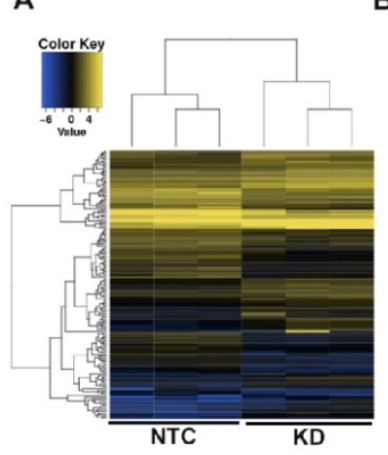

C
B

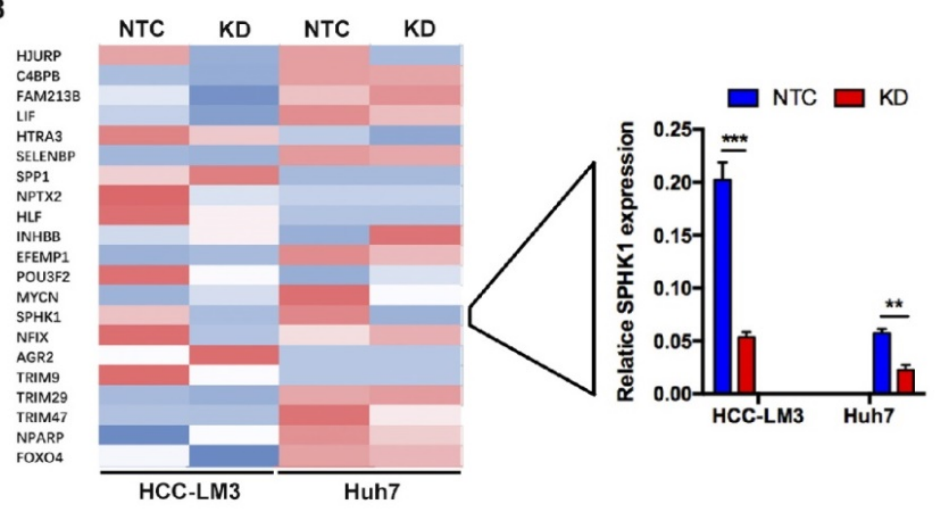

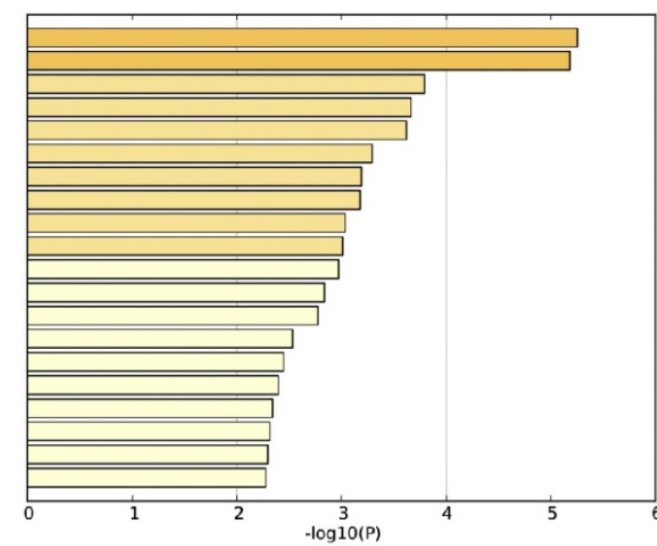

M2772: ESC V6.5 UP LATE.V1 UP M2725: MEK UP.V1 UP

GO:0055123: digestive system development

GO:0030198: extracellular matrix organization

GO:1903035: negative regulation of response to wounding

R-HSA-109582: Hemostasis

M2816: RPS14 DN.V1 UP

M2682: ATF2 S UP.V1 UP

GO:0030170: pyridoxal phosphate binding

GO:0072523: purine-containing compound catabolic process

GO:0043687: post-translational protein modification

GO:0004714: transmembrane receptor protein tyrosine kinase activity

M3468: NABA ECM REGULATORS

GO:0006665: sphingolipid metabolic process *

GO:0032414: positive regulation of ion transmembrane transporter activity

GO:0007389: pattern specification process

M2824: II 21 UP V1 DN

M2824: IL21 UP.V1 DN

M2723: LTE2 UP.V1 UP

M2728: RAF UP.V1 UP

D

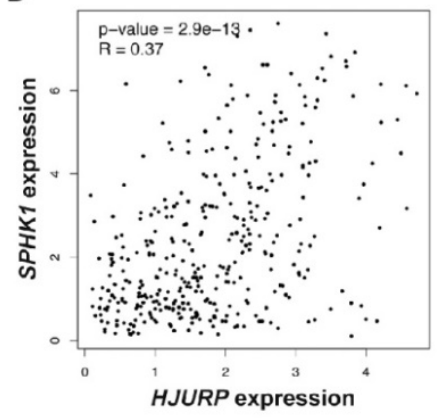

E

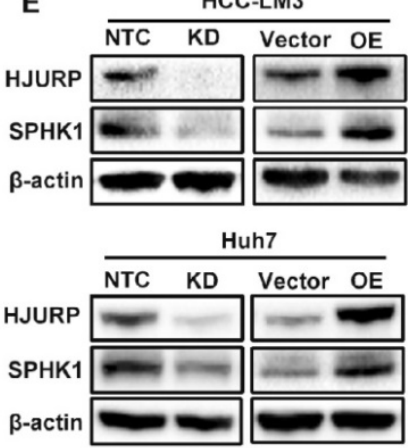

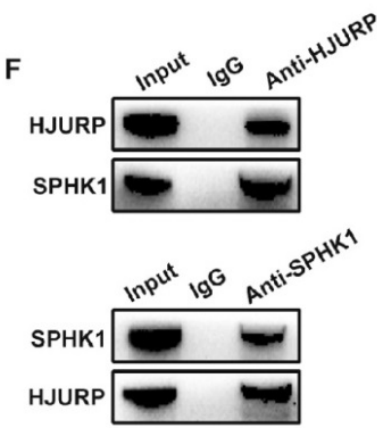

Figure 3. HJURP facilitates EMT via upregulating Sphingosine Kinase 1. (A) Microarray analysis of NTC and KD group in Huh7 cells. (B) EMT-related genes were analyzed by RT-qPCR in HCC-LM3 and Huh7 cells. Relative mRNA levels of 20 genes (left) and SPHK1 (right) are shown. (C) KEGG (Kyoto Encyclopedia of Genes and Genomes) pathways enriched in 164 genes whose expression is correlated with HJURP. The sphingolipid metabolic progress is marked with "**. (D) Scatter plots shows a positive correlation between HJURP and SPHK1 at the mRNA level. Data were from TCGA database. (E) The protein level of HJURP and SPHK1 were detected by western-blot in HCC-LM3 and Huh7 cells. (F) Co-Immunoprecipitation assay shows HJURP interacts with SPHK1 in HCC-LM3 cells. 
A

NTC

KD

KD+SP

Huh7
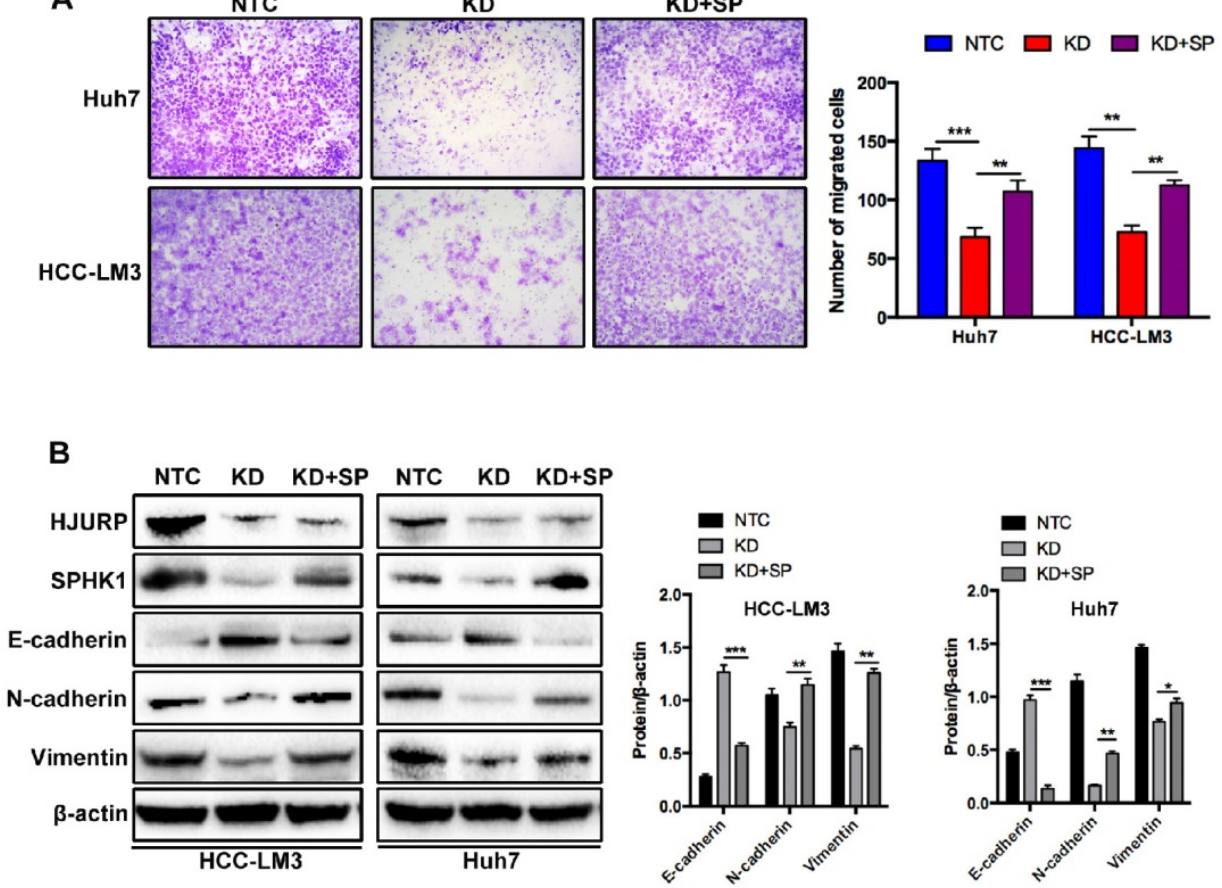

Figure 4. The effects of SPHK1 overexpression on HJURP induced EMT. (A) Compared to HJURP KD group, SPHK1 overexpression (KD+SP) significantly enhanced the invasive ability in HJURP-knockdown Huh7 and HCC-LM3 cells. (B) SPHK1 overexpression in HJURP knockdown group increased the expression of E-cadherin but decreased the expression of $\mathrm{N}$-cadherin and Vimentin.

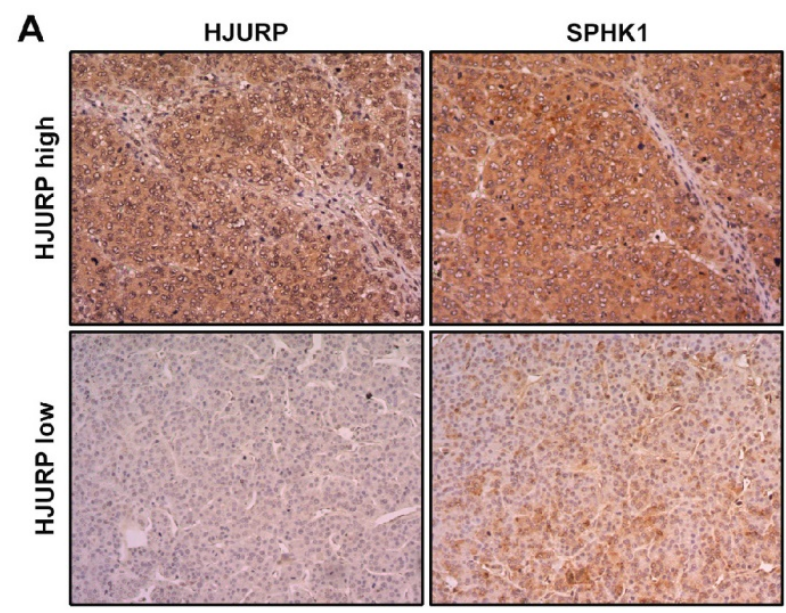

B

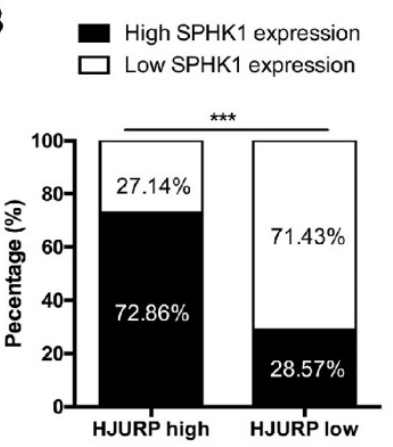

C

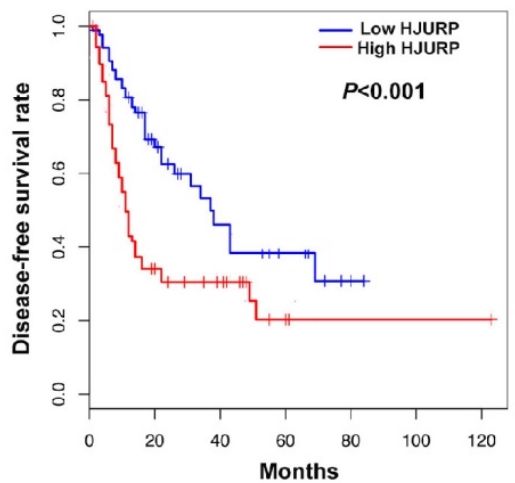

Figure 5. The expression of HJURP is positively correlated with SPHK1 and high HJURP expression predicts poor disease-free survival rate of HCC patients. (A) and (B) Immunohistochemical staining of HCC tissues showed that high HJURP expression is correlated with high SPHK1 expression (magnification: 200X). (C) Kaplan-Meier analysis showed that HCC patients with low HJURP expression had a better disease-free survival rate than patients with high HJURP expression. 
Table 2. Relation between HJURP expression and clinicopathological features in HCC patients.

\begin{tabular}{llll}
\hline & \multicolumn{3}{c}{ HJURP expression } \\
\hline $\begin{array}{l}\text { Clinicopathological features } \\
\text { Sex }\end{array}$ & Low(n=49) & High(n=70) & P value \\
$\begin{array}{l}\text { Male(n=105) } \\
\text { Female(n=14) }\end{array}$ & 45 & 60 & 0.31 \\
$\begin{array}{l}\text { Age(years) } \\
>65(n=22)\end{array}$ & 4 & 10 & \\
$\leq 65(n=97)$ & 13 & 9 & 0.06 \\
HBV & 36 & 61 & \\
Positive(n=102) & & & 0.29 \\
Negative(n=17) & 40 & 62 & \\
PVTT & 9 & 8 & \\
Yes(n=29) & & & \\
No(n=90) & 8 & 21 & \\
MVI & 41 & 49 & \\
Yes(n=51) & & & \\
No(n=68) & 15 & 36 & \\
Recurrence & 34 & 34 & \\
Yes(n=59) & & & \\
No(n=60) & 26 & 33 & \\
Encapsulation & 23 & 37 & \\
Yes(n=51) & & & \\
No(n=68) & 22 & 29 & \\
\hline
\end{tabular}

PVTT: portal vein tumor thrombus; MVI: microvascular invasion. $P$ value marked with "*" are statistically significant.

Table 3. Multivariable cox analysis of tumor recurrence.

\begin{tabular}{lll}
\hline Variables & P value & HR(95\%CI) \\
\hline Sex & 0.156 & $0.462(0.154-1.381)$ \\
Age & 0.692 & $0.994(0.969-1.021)$ \\
HBV & 0.671 & $1.206(0.616-2.361)$ \\
PVTT & 0.766 & $0.593(0.121-2.901)$ \\
MVI & $<0.001^{*}$ & $7.050(3.626-13.705)$ \\
Encapsulation & 0.444 & $0.736(0.317-1.708)$ \\
HJURP & $0.038^{*}$ & $1.945(1.007-3.745)$ \\
\hline \multicolumn{2}{l}{ HR: risk ratio; 95\%CI: 95\% confidence interval. P value marked with “*” are } \\
statistically significant.
\end{tabular}

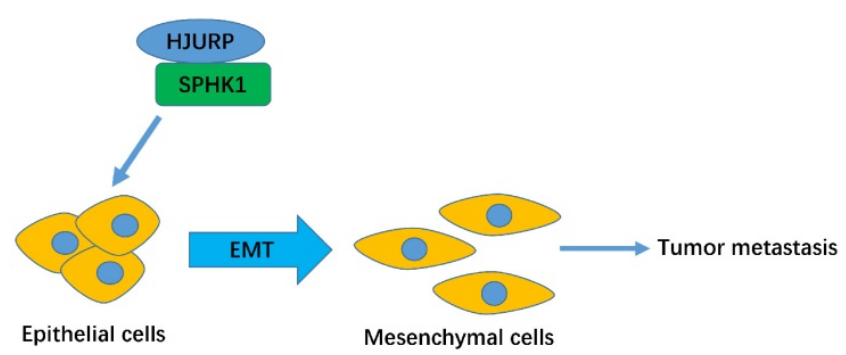

Figure 6. The schematic model of HJURP-induced metastasis in HCC.

SPHK1 is a regulator of sphingosine metabolites, which can facilitate the development of human neoplasms including HCC. Zhang and Bao et.al. reported that SPHK1 overexpression facilitates the migration and invasion in SMMC-7721 cells[23, 24]. We found that the expression of SPHK1 is positively correlated with HJURP both in HCC cells and tissues. And the KEGG analysis revealed HJURP could mediate the sphingolipid metabolite process, so we conjectured that HJURP promotes EMT via SPHK1.
Besides, SPHK1 overexpression in HJURP-knockdown cells reversed the invasion abilities and the expression of EMT markers in HCC-LM3 and Huh7 cells. Given that HJURP facilitates EMT by interacting with SPHK1 (as shown in Co-IP assay), the underlying mechanism involved in SPHK1 mediated EMT in HCC remains interesting. Although we did not research it in this work, it has been clarified in Liu's research[18]. They revealed that SPHK1 promotes the EMT by stimulating autophagy and then accelerating the lysosomal degradation of the epithelial marker E-cadherin in HCC cells. According to current reports and our results, we consider that HJURP can promote EMT via SPHK1.

We have reported that high expression of HJURP predicts lower overall survival rate of HCC patients[8]. In this work, we found that high HJURP expression positively correlated with EMT and microvascular invasion in HCC individuals. As microvascular invasion is a major step in the process of tumor metastasis, we consider that those two findings consistent with each other.

In conclusion, we demonstrated that HJURP regulated EMT via upregulating SPHK1. In addition, high HJURP expression predicts a lower disease-free survival rate and higher possibility of microvascular invasion.

\section{Abbreviations}

HJURP: Holliday Junction Recognition Protein; HCC: Hepatocellular carcinoma; SPHK1: Sphingosine kinase1; EMT: Epithelial-to-Mesenchymal Transition; CO-IP: Co-Immunoprecipitation.

\section{Supplementary Material}

Supplementary figures and tables. http://www.ijbs.com/v15p1139s1.pdf

\section{Acknowledgement}

This research was funded by National S\&T Major Project (No. 2017ZX10203205-003-002), Innovative Research Groups of National Natural Science Foundation of China (No.81721091) and Zhejiang International Science and Technology Cooperation Project (No.2016C04003).

\section{Author Contributions}

Conceptualization: Tianchi Chen and Shusen Zheng; Methodology: Tianchi Chen, Wuhua Zhou, Hechen Huang and Shengyong Yin; Investigation: Tianchi Chen, Lingfeng Zhou and Yuan Zhou; Writing of the manuscript: Tianchi Chen and Lingfeng Zhou. 


\section{Ethics Committee Approval and Patient Consent}

All clinical tissues used in this experiment were from the First Affiliated Hospital, Zhejiang University School of Medicine, Zhejiang, China. The research was approved by the Ethical Review Committee of this hospital. And informed consent was obtained according to the guidelines of the Declaration of Helsinki. All experiments also have been conducted in accordance with relevant national legislation on the use of animals for research.

\section{Competing Interests}

The authors have declared that no competing interest exists.

\section{References}

1. Torre LA, Bray F, Siegel RL, Ferlay J, Lortet-Tieulent J, Jemal A. Global cancer statistics, 2012. CA Cancer J Clin. 2015; 65: 87-108.

2. Kong Y, Zhang L, Huang Y, He T, Zhang L, Zhao X, et al. Pseudogene PDIA3P1 promotes cell proliferation, migration and invasion, and suppresses apoptosis in hepatocellular carcinoma by regulating the p53 pathway. Cancer Lett. 2017; 407: 76-83.

3. Wong R, Frenette C. Updates in the management of hepatocellular carcinoma. Gastroenterol Hepatol (N Y). 2011; 7: 16-24.

4. El-Serag HB, Marrero JA, Rudolph L, Reddy KR. Diagnosis and treatment of hepatocellular carcinoma. Gastroenterology. 2008; 134: 1752-63.

5. Barnhart-Dailey MC, Trivedi P, Stukenberg PT, Foltz DR. HJURP interaction with the condensin II complex during G1 promotes CENP-A deposition. Mol Biol Cell. 2017; 28: 54-64.

6. Muller S, Montes de Oca R, Lacoste N, Dingli F, Loew D, Almouzni G. Phosphorylation and DNA binding of HJURP determine its centromeric recruitment and function in CenH3(CENP-A) loading. Cell reports. 2014; 8: 190-203.

7. Hu B, Wang Q, Wang Y, Chen J, Li P, Han M. Holliday junction-recognizing protein promotes cell proliferation and correlates with unfavorable clinical outcome of hepatocellular carcinoma. Onco Targets Ther. 2017; 10: 2601-7.

8. Chen T, Huang H, Zhou Y, Geng L, Shen T, Yin S, et al. HJURP promotes hepatocellular carcinoma proliferation by destabilizing p21 via the MAPK/ERK1/2 and AKT/GSK3beta signaling pathways. J Exp Clin Cancer Res. 2018; 37: 193.

9. Huang $W$, Zhang $H$, Hao $Y, X u X$, Zhai $Y$, Wang $S$, et al. A Non-Synonymous Single Nucleotide Polymorphism in the HJURP Gene Associated with Susceptibility to Hepatocellular Carcinoma among Chinese. PloS one. 2016; 11: e0148618.

10. Acloque $\mathrm{H}$, Adams MS, Fishwick $\mathrm{K}$, Bronner-Fraser $\mathrm{M}$, Nieto MA. Epithelial-mesenchymal transitions: the importance of changing cell state in development and disease. J Clin Invest. 2009; 119: 1438-49.

11. Thiery JP, Acloque H, Huang RY, Nieto MA. Epithelial-mesenchymal transitions in development and disease. Cell. 2009; 139: 871-90.

12. Chapman HA. Epithelial-mesenchymal interactions in pulmonary fibrosis. Annu Rev Physiol. 2011; 73: 413-35.

13. Thiery JP, Sleeman JP. Complex networks orchestrate epithelial-mesenchymal transitions. Nat Rev Mol Cell Biol. 2006; 7: 131-42.

14. Yang J, Mani SA, Donaher JL, Ramaswamy S, Itzykson RA, Come C, et al. Twist, a master regulator of morphogenesis, plays an essential role in tumor metastasis. Cell. 2004; 117: 927-39.

15. Nawshad A, Hay ED. TGFbeta3 signaling activates transcription of the LEF1 gene to induce epithelial mesenchymal transformation during mouse palate development. J Cell Biol. 2003; 163: 1291-301.

16. Baker DL, Pham TC, Sparks MA. Structure and catalytic function of sphingosine kinases: analysis by site-directed mutagenesis and enzyme kinetics. Biochim Biophys Acta. 2013; 1831: 139-46.

17. Xu CY, Liu SQ, Qin MB, Zhuge CF, Qin L, Qin N, et al. SphK1 modulates cell migration and EMT-related marker expression by regulating the expression of p-FAK in colorectal cancer cells. Int J Mol Med. 2017; 39: 1277-84.

18. Liu H, Ma Y, He HW, Zhao WL, Shao RG. SPHK1 (sphingosine kinase 1) induces epithelial-mesenchymal transition by promoting the autophagy-linked lysosomal degradation of $\mathrm{CDH} 1 /$ E-cadherin in hepatoma cells. Autophagy. 2017; 13: 900-13.

19. Lambert AW, Pattabiraman DR, Weinberg RA. Emerging Biological Principles of Metastasis. Cell. 2017; 168: 670-91.

20. Yilmaz M, Christofori G. EMT, the cytoskeleton, and cancer cell invasion. Cancer Metastasis Rev. 2009; 28: 15-33.
21. Priya R, Yap AS. Making a Choice: How Cadherin Switching Controls Cell Migration. Dev Cell. 2015; 34: 383-4.

22. Tarhan YE, Kato T, Jang M, Haga Y, Ueda K, Nakamura $Y$, et al. Morphological Changes, Cadherin Switching, and Growth Suppression in Pancreatic Cancer by GALNT6 Knockdown. Neoplasia. 2016; 18: 265-72.

23. Zhang Z, Yan Z, Yuan Z, Sun Y, He H, Mai C. SPHK1 inhibitor suppresses cell proliferation and invasion associated with the inhibition of NF-kappaB pathway in hepatocellular carcinoma. Tumour Biol. 2015; 36: 1503-9.

24. Bao $\mathrm{M}, \mathrm{Chen} \mathrm{Z}, \mathrm{Xu} \mathrm{Y}, \mathrm{Zhao} \mathrm{Y}, \mathrm{Zha} \mathrm{R}$, Huang S, et al. Sphingosine kinase 1 promotes tumour cell migration and invasion via the S1P/EDG1 axis in hepatocellular carcinoma. Liver Int. 2012; 32: 331-8. 